\title{
DOBUTAMINE STRESS ECHOCARDIOGRAPHY FOR THE PREOPERATIVE EVALUATION OF PATIENTS UNDERGOING LUNG VOLUME REDUCTION SURGERY
}

Eduardo Bossone, $\mathrm{MD}, \mathrm{PhD}^{\mathrm{a}}$

Fernando J. Martinez, MD

Richard I. Whyte, $\mathrm{MD}^{\mathrm{c}}$

Mark D. Iannettoni, $\mathrm{MD}^{\mathrm{c}}$

William F. Armstrong, MD

David S. Bach, MD
Background: Lung volume reduction surgery has been proposed as a bridge to lung transplantation and as definitive therapy for advanced chronic obstructive lung disease. However, patient selection criteria and optimal preoperative assessment have not been clearly defined. Objective: We investigated the feasibility, safety, and value of dobutamine stress echocardiography as a predictor of major early cardiac events in patients who underwent lung volume reduction surgery. Methods: The study population consisted of 46 patients ( 21 men and 25 women, mean age $59 \pm 9$ years) who underwent dobutamine stress echocardiography (maximum dose $40 \mu \mathrm{g} \cdot \mathrm{kg}^{-1} \cdot \mathrm{min}^{-1}$ plus atropine if needed) 180 days or less before lung volume reduction surgery. Adverse cardiac events were prospectively defined and tabulated during hospitalization after the operation and at subsequent outpatient visits. Results: Dobutamine stress echocardiography was interpretable in 45 of $46(98 \%)$ patients. There were no adverse events during testing. The studies revealed normal left ventricular systolic function at rest in all patients and normal right ventricular function in all patients but one. Thirteen patients had right ventricular enlargement. Estimated right ventricular systolic pressure was mildly elevated (>40 mm Hg) in 5 patients. Four patients (9\%) had stress tests positive for ischemia. There were no perioperative deaths. Follow-up was available for 44 of 45 patients at a duration of $20.0 \pm 7.0$ months. Two major adverse cardiac events occurred in the same patient in whom the results of dobutamine stress echocardiography were positive for ischemia (positive predictive value $25 \%, 95 \%$ confidence interval $0 \%$ to $83 \%$; negative predictive value $100 \%, 95 \%$ confidence interval 90 to $100 \%)$. Conclusion: Despite end-stage chronic obstructive lung disease and poor ultrasound windows, dobutamine stress echocardiography is feasible and safe in patients undergoing evaluation for lung volume reduction surgery. It yields important information on right and left ventricular function and has an excellent negative predictive value for early and late adverse cardiac events. (J Thorac Cardiovasc Surg 1999;118:542-6) ung volume reduction surgery (LVRS) is currently undergoing evaluation as a bridge to lung transplantation and as definitive therapy for advanced chronic obstructive lung disease. ${ }^{1}$ However, the prevalence of coronary artery disease (CAD) and of pulmonary hypertension among patients with a history of tobacco use and severe obstructive lung disease are associated

From the Department of Internal Medicine, Divisions of Cardiology, ${ }^{\mathrm{a}}$ and Pulmonary and Critical Care Medicine, ${ }^{b}$ and the Department of Surgery, Section of Thoracic Surgery, ${ }^{\mathrm{C}}$ University of Michigan, Ann Arbor, Mich.

Received for publication Oct 9, 1998; revisions requested Feb 19, 1999; revisions received April 5, 1999; accepted for publication May 26, 1999. with a high risk of cardiovascular morbidity among an unselected population undergoing LVRS. ${ }^{2}$ Although there is agreement that patients should be evaluated preoperatively for high-risk cardiovascular disease, ${ }^{1,2}$ there is currently no consensus with respect to specific preoperative testing before LVRS.

Dobutamine stress echocardiography (DSE) is an

\footnotetext{
Address for reprints: David S. Bach, MD, University of Michigan, L3119 Women's-0273, 1500 E. Medical Center Dr, Ann Arbor, MI 48109-0273 (E-mail:dbach@umich.edu).

Copyright $\odot 1999$ by Mosby, Inc.

$0022-5223 / 99 \$ 8.00+0 \quad \mathbf{1 2 / 1 / 1 0 0 2 7 6}$
} 
accepted means for the evaluation of inducible ischemia ${ }^{3,4}$ and for the assessment of perioperative risk. ${ }^{5-7}$ In addition, echocardiography can be used to assess both left and right ventricular systolic function, evaluate the presence and severity of valvular heart disease, and estimate right ventricular systolic pressure. ${ }^{8,9}$ The presence of severe obstructive lung disease may compromise echocardiographic windows and may result in suboptimal image quality in this population. The purpose of the present study was to evaluate the feasibility, safety, and prognostic value of DSE in a group of patients undergoing LVRS.

\section{Methods}

Study population. The study population consisted of 46 consecutive patients who underwent DSE at the University of Michigan 180 days or less before LVRS during 1995 and 1996. The average time between DSE and LVRS was $77 \pm 41$ days (range 8-179 days).

Cardiac risk profile. Risk factors for CAD or a known history of CAD were prospectively investigated during preoperative clinical assessment. Risk factors included family history of CAD, systemic hypertension, diabetes mellitus, hypercholesterolemia, and recent tobacco use. Of note, nearly all patients had a history of tobacco use at some time in the past, but it was reported only if recent.

Imaging protocol. Echocardiographic imaging was performed with standard, commercially available equipment. Imaging included visualization of left- and right-sided cardiac chambers and valves for evaluation of left and right ventricular size and function, as well as for evidence of valvular heart disease. In addition to the assessment of ventricular function and valvular heart disease at baseline, right ventricular systolic pressure was estimated from the spectral profile of the tricuspid regurgitation jet. The right ventricular systolic pressure was calculated by adding a fixed value of $14 \mathrm{~mm} \mathrm{Hg}$ (assumed right atrial pressure) to the systolic transtricuspid gradient $\left(\Delta \mathrm{P}=4 \times \mathrm{V}^{2}\right.$, where $\mathrm{V}=$ maximum velocity of tricuspid regurgitation). ${ }^{8-11}$ In accordance with routine laboratory practice, contrast injection was not routinely used for enhancement of the tricuspid regurgitation envelope. Pulmonary hypertension was defined as any right ventricular systolic pressure greater than $40 \mathrm{~mm} \mathrm{Hg}$.

Dobutamine protocol. DSE was performed as previously described. ${ }^{4}$ Antianginal medications were not withheld before testing. Windows were used during echocardiographic imaging to optimize visualization of left ventricular wall motion and systolic function. Imaging typically included parasternal long- and short-axis, apical 4- and 2-chamber, and subcostal views. Dobutamine was infused in 3-minute stages of 10, 20, 30 , and $40 \mu \mathrm{g} \cdot \mathrm{kg}^{-1} \cdot \mathrm{min}^{-1}$. Atropine was administered in $0.25 \mathrm{mg}$ boluses up to $1.0 \mathrm{mg}$ total dose if the heart rate was less than 100 beats/min at $30 \mu \mathrm{g} \cdot \mathrm{kg}^{-1} \cdot \mathrm{min}^{-1}$. Blood pressure, 12-lead electrocardiograms, and echocardiograms were recorded during each stage and at 10 minutes of recovery. All echocardiographic images were recorded on standard VHS videotape. In addition, echocardiographic images at baseline, low stress $\left(10 \mathrm{mg} \cdot \mathrm{kg}^{-1} \cdot \mathrm{min}^{-1}\right)$, peak stress, and recovery were digitized and displayed in quad-screen format for subsequent off-line analysis. The left ventricle was analyzed with the use of a 16-segment model. ${ }^{12,13}$ Wall motion score index (WMSI) was calculated at rest and peak dobutamine stress with the use of a standard formula in which normal $=1$, hypokinesia $=2$, akinesia $=3$, and dyskinesia $=4$. Ischemia was defined as the development of a new or worsening wall motion abnormality during stress. Tests were interpreted by experienced echocardiographers at the time of performance. Reviewers were blinded to clinical information and to the results of any other cardiac tests. The protocol for DSE was reviewed and approved by the University of Michigan Institutional Review Board. All patients provided written informed consent.

Clinical follow-up. Patients were monitored during hospitalization and returned after discharge for regular follow-up visits. Cardiac events were prospectively defined on the basis of standard clinical definitions, recorded at the time of occurrence and subsequently compiled by retrospective review. Major cardiac events were considered to be cardiac death, nonfatal myocardial infarction, congestive heart failure, and unstable angina pectoris necessitating hospitalization. Minor cardiac events included atrial fibrillation, other supraventricular tachycardia, or hypotension.

Statistics. All data are presented as mean \pm 1 standard deviation. Comparison of hemodynamics at baseline and peak stress were made by means of the paired Student $t$ tests.

\section{Results}

Study population demographics are outlined in Table I. At least 1 risk factor for CAD was present in 34 patients (74\%), with 2 or more risk factors present in 12 patients (26\%). Tobacco use was by far the most prevalent risk factor, followed by hypertension, known CAD, and then hyperlipidemia and family history of CAD.

Feasibility and safety. Of 46 patients who underwent both DSE and subsequent LVRS, 45 (98\%) had echocardiography/Doppler images adequate for analysis. Assessment of left ventricular wall motion for ischemia was performed in all of the 45 patients who underwent DSE.

DSE was well tolerated. The most frequent side effects were minor and included palpitations in 38 patients (84\%), of whom 11 (29\%) had shortness of breath and 1 had nausea. No patient had angina, significant ventricular or supraventricular arrhythmias, or myocardial infarction.

Resting echocardiography. Of 45 echocardiographic examinations, 19 (42\%) were completely normal with no evidence of valve disease and normal left- and right-sided chamber size and function. A total of 43 
Table I. Population demographics

\begin{tabular}{llll}
\hline & \multicolumn{1}{c}{ Total } & Unilateral & Bilateral \\
\hline LVRS & 46 & $7(15 \%)$ & $39(85 \%)$ \\
Age (y) & & & \\
$\quad$ Mean \pm SD (range) & $59 \pm 9(38-74)$ & $50 \pm 11$ & $61 \pm 7$ \\
Sex (M/F) & $21 / 25(46 \% / 54 \%)$ & $4 / 3$ & $18 / 21$ \\
Diagnosis & & & \\
$\quad$ Emphysema & $45(98 \%)$ & 6 & 39 \\
A1AT & $1(2 \%)$ & 1 & 0 \\
CAD risk factors & & & \\
Recent tobacco use & $30(65 \%)$ & & \\
Hypertension & $9(19 \%)$ & & \\
Cholesterol > 200 & $1(2 \%)$ & & \\
$\quad$ mg/dL & $1(2 \%)$ & & \\
Family history of & $\quad$ & \\
$\quad$ CAD & 0 & \\
Diabetes & $4(9 \%)$ & \\
Known CAD &
\end{tabular}

AlAT, Alpha-1 antitrypsin deficiency; LVRS, lung volume reduction surgery.

Table II. Resting 2-dimensional echocardiographic abnormalities

\begin{tabular}{lcr}
\hline & No. & $\%$ \\
\hline Dilated left ventricle & 2 & 4 \\
Dilated right ventricle & 13 & 29 \\
Dilated left atrium & 4 & 9 \\
Dilated right atrium & 9 & 20 \\
Left ventricular hypertrophy & 1 & 2 \\
Right ventricular hypertrophy & 2 & 4 \\
Wall motion abnormalities at rest & 2 & 4 \\
Right ventricular dysfunction & 1 & 2 \\
Flattened septum with pressure overload & 8 & 18 \\
Valvular heart disease & 1 & 2 \\
\hline
\end{tabular}

echocardiographic or Doppler abnormalities were noted among the remaining 26 patients, of which 33 (78\%) were associated with the right side of the heart and $10(23 \%)$ with the left side of the heart. The Doppler signal of tricuspid regurgitation was adequate for the estimation of right ventricular systolic pressure in $35(76 \%)$ patients, of whom $30(86 \%)$ had a right ventricular systolic pressure of $40 \mathrm{~mm} \mathrm{Hg}$ or less and 5 (14\%) had a pressure of more than $40 \mathrm{~mm} \mathrm{Hg}$. Of the 5 patients with a right ventricular systolic pressure of more than $40 \mathrm{~mm} \mathrm{Hg}$, the average estimated pressure was $56 \pm 12 \mathrm{~mm} \mathrm{Hg}$ (range 41-70 mm Hg). Mild pulmonary hypertension was confirmed on right heart catheterization in all 5 of these patients (pulmonary artery systolic pressure $41.8 \pm 5.4 \mathrm{~mm} \mathrm{Hg}$ ). Table II summarizes the 2-dimensional echocardiographic abnormalities noted among all patients.

DSE. The hemodynamic changes with dobutamine are shown in Table III. Two patients had abnormal wall
Table III. Hemodynamic changes with dobutamine

\begin{tabular}{|c|c|c|c|c|}
\hline & Baseline & Peak & $\Delta$ & $\mathrm{P}$ value \\
\hline \multicolumn{5}{|l|}{$\begin{array}{l}\text { Heart rate } \\
\text { (beats/min) }\end{array}$} \\
\hline Mean \pm SD & $78 \pm 13$ & $121 \pm 15$ & $43.3 \pm 13.2$ & $<.001$ \\
\hline Range & 51 to 111 & 85 to 170 & 19 to 88 & \\
\hline \multicolumn{5}{|c|}{$\begin{array}{l}\text { Systolic blood } \\
\text { pressure }(\mathrm{mm} \mathrm{Hg})\end{array}$} \\
\hline Mean \pm SD & $132 \pm 13$ & $128 \pm 18$ & $-5.2 \pm 18.7$ & .1 \\
\hline Range & 106 to 159 & 91 to 172 & -43 to +35 & \\
\hline \multicolumn{5}{|c|}{$\begin{array}{l}\text { Diastolic blood } \\
\text { pressure }(\mathrm{mm} \mathrm{Hg})\end{array}$} \\
\hline Mean \pm SD & $73 \pm 9$ & $65 \pm 15$ & $-7.6 \pm 15.4$ & .001 \\
\hline Range & 56 to 94 & 43 to 107 & -44 to +40 & \\
\hline
\end{tabular}

motion at baseline. For these 2 patients, WMSI at baseline was 2.09 and 2.31, respectively. DSE revealed inducible ischemia in 4 of 45 patients (9\%), including both patients with abnormal wall motion at baseline. For the 2 patients with abnormal wall motion at baseline, WMSI increased with dobutamine from 2.09 and 2.31 to 2.28 and 2.63 , respectively. WMSI for the 4 patients with inducible ischemia increased from $1.6 \pm$ 0.7 (range 1.0-2.3) to $1.8 \pm 0.7$ (range 1.1-2.6), with stress $(\Delta=0.2 \pm 0.1$; range $0.1-0.3)$. For the remaining 42 patients, WMSI remained 1.0 at peak stress (normal response).

Outcome. Clinical data were available for all patients during their hospitalization after the operation. The average length of hospital stay after LVRS was 11 \pm 6 days (range 5-25 days). No patient died during the operation or during the subsequent hospitalization. Similarly, no patients had acute myocardial infarction or unstable angina in the operative or perioperative period. A single episode of pulmonary edema occurred in 1 of the 4 patients with ischemia on DSE. Two other patients had minor cardiac events. One patient with normal DSE test results had paroxysmal atrial fibrillation develop; another patient with abnormal DSE test results had episodes of supraventricular tachycardia with hypotension. Both arrhythmias resolved with appropriate therapy. On the basis of the single major event among 4 patients with ischemia on DSE, there was a positive predictive value for DSE in predicting major perioperative events of $25 \%$ (95\% CI $0 \%-83 \%$ ). No patient with a negative DSE test result had any major cardiac complication, yielding a negative predictive value for major cardiac events of $100 \%$ (95\% CI $90 \%-100 \%)$.

Follow-up after discharge was available for 44 of 45 patients (98\%) at a duration of $20.0 \pm 7.0$ months (range 4-31 months) after the operation. Follow-up was 
Table IV. Outcome for patients with abnormal DSE or adverse cardiac events

\begin{tabular}{|c|c|c|c|c|c|c|c|c|c|c|}
\hline \multirow{3}{*}{$\begin{array}{l}\text { Patient } \\
\text { (age }[y], \text { sex) }\end{array}$} & \multirow{3}{*}{$\begin{array}{c}\text { Risk factors } \\
\text { for } C A D\end{array}$} & \multirow[b]{3}{*}{$L V R S$} & \multirow{3}{*}{$\begin{array}{l}\text { Hospital } \\
\text { stay }(d)\end{array}$} & \multirow{3}{*}{$\begin{array}{l}\text { Follow-up } \\
\qquad(d)\end{array}$} & \multirow{3}{*}{$\begin{array}{c}\text { Preop RVSP } \\
\text { (mm } \mathrm{Hg})\end{array}$} & \multicolumn{3}{|c|}{$D S E$} & \multirow{2}{*}{\multicolumn{2}{|c|}{ Cardiac events }} \\
\hline & & & & & & WMSI & WMSI & WMSI & & \\
\hline & & & & & & Base & Peak & $\Delta$ & Early & Late \\
\hline $1(64, M)$ & Tob/HTN & B & 14 & 577 & $<40$ & 1 & 1 & 0 & 0 & A Flutter \\
\hline $2(58, F)$ & Tob & B & 8 & 556 & $<40$ & 1 & 1 & 0 & A Fib & A Fib \\
\hline $3(59, F)$ & Tob/HTN & B & 24 & 810 & $<40$ & 2.09 & 2.28 & 0.19 & $\mathrm{CHF}$ & $\mathrm{CHF}$ \\
\hline $4(70, M)$ & Tob/HTN/HL & B & 10 & 810 & $<40$ & 1 & 1.06 & 0.06 & SVT/Нypo & 0 \\
\hline $5(59, \mathrm{~F})$ & CAD & $\mathrm{U}$ & 13 & 296 & $<40$ & 2.31 & 2.63 & 0.32 & 0 & 0 \\
\hline $6(43, F)$ & Tob/HTN & $\mathrm{U}$ & 5 & 506 & $<40$ & 1 & 1.31 & 0.31 & 0 & 0 \\
\hline
\end{tabular}

A Fib, Atrial fibrillation; A Flutter, atrial flutter; $B$, bilateral; $C H F$, congestive heart failure; $H L$, hyperlipidemia; $H T N$, hypertension; $R V S P$, right ventricular systolic pressure (from echo); Tob, recent tobacco use; SVT/Hypo, supraventricular tachycardia with hypotension; $U$, unilateral; WMSI, wall motion score index.

6 months or more for 43 of 45 patients $(96 \%)$ and more than 1 year for 39 of $45(87 \%)$. During long-term follow-up there were no major cardiac events. One episode of pulmonary edema and 1 episode of paroxysmal atrial fibrillation occurred in the same 2 patients who experienced these complications during hospitalization. One additional patient with a negative DSE test result had atrial flutter in the follow-up period. Table IV summarizes DSE results and prognosis for those patients with either an abnormal DSE suggestive of ischemia or any early or late cardiac event.

\section{Discussion}

DSE is an accepted technique for the preoperative assessment of cardiac risk in patients undergoing noncardiac surgery. ${ }^{14-23}$ Several studies have demonstrated the feasibility, safety, and prognostic value of DSE for perioperative and late cardiac events in both vascular surgery ${ }^{14-22}$ and general surgical procedures. ${ }^{23}$ Because candidates for LVRS are frequently former heavy smokers with a presumed high prevalence of cor pulmonale and $\mathrm{CAD},{ }^{2}$ surgical morbidity has been found to be prohibitive in unselected populations. ${ }^{1,24,25}$ Limitations in exercise capacity resulting from underlying advanced lung disease make pharmacologic stress testing an ideal tool to assess these patients for occult CAD and inducible ischemia. Although nuclear scintigraphy allows assessment for CAD, echocardiography also allows for the assessment of left and right ventricular systolic function, valvular heart disease, and evidence of pulmonary hypertension. However, echocardiographic windows are affected by hyperexpansion of lung parenchyma, and acoustic windows may be limited in this patient population. The present study is the first to our knowledge to address the feasibility and the prognostic power of DSE in a surgical population limited to patients with end-stage obstructive lung disease undergoing a major intrathoracic procedure.
Feasibility and safety. In the present study, DSE was feasible in $98 \%(45 / 46)$ of patients, despite the presence of end-stage lung disease in all of them. Even though these patients had significant comorbid disease, DSE was found to have a safety profile similar to that noted in other patient populations. ${ }^{14-23}$

Predictive power. DSE was found to have an excellent negative predictive value for early and late cardiac events, which appears to be comparable with that found for other types of noncardiac surgery. ${ }^{14-23}$ In particular, for patients undergoing vascular surgery, the negative predictive value of dobutamine-atropine stress echocardiography for perioperative and late cardiac events ranges from $95 \%$ to $100 \% .^{7}$ A high negative predictive value of $94 \%$ was found as well by Bates and associates $^{23}$ in patients with insulin-dependent diabetes mellitus before kidney and/or pancreas transplantation.

Limitations. Very few adverse cardiac events and no major adverse events occurred in the surgical population. However, this finding is reflective of the highly selected nature of this population and the fact that physicians were not blinded to the results of DSE. Patients with clinically apparent cardiovascular abnormalities likely were not referred for subsequent surgery. This is not a unique finding and it is in accordance with the very low rate of cardiac complications demonstrated by previous investigators in similar studies after patients have been appropriately screened for surgery. ${ }^{2}$ In a study by Cooper and colleagues, ${ }^{26}$ only 4 of $150(2.6 \%)$ consecutive patients had major cardiac complications after bilateral LVRS, including 2 with myocardial infarction and 2 with cardiac arrest. Similarly, McKenna and coworkers ${ }^{27}$ found only 1 major in-hospital cardiac event after LVRS among 166 consecutive patients $(0.6 \%)$. As noted earlier, the low rate of perioperative events among the present and previous populations undergoing LVRS was likely due to application of conservative selection criteria. 


\section{Conclusion}

DSE is safe and feasible for the preoperative assessment of cardiac risk among candidates for LVRS. It represents a highly versatile diagnostic tool that provides important information regarding left and right ventricular systolic function, valvular heart disease, and presence of CAD and inducible ischemia. A negative DSE test result implies low perioperative and subsequent cardiac risk among patients referred for LVRS.

\section{REFERENCES}

1. Weinmann GG, Hyatt R. Evaluation and research in lung volume reduction surgery. Am J Respir Crit Care Med 1996;154:1913-8.

2. Thurnheer R, Muntwjler J, Stammberger U, Bloch KE, Zollinger A, Weder W, et al. Coronary artery disease in patients undergoing lung volume reduction surgery for emphysema. Chest 1997;112: 112-28.

3. Geleijnse ML, Fioretti PM, Roelandt JRTC. Methodology, feasibility, safety and diagnostic accuracy of dobutamine stress echocardiography. J Am Coll Cardiol 1997;30:595-606.

4. Marcovitz PA, Armstrong WF. Accuracy of dobutamine stress echocardiography in detecting coronary artery disease. Am J Cardiol 1992;69:1269-73.

5. Guidelines for perioperative cardiovascular evaluation for noncardiac surgery: report of the American College of Cardiology/ American Heart Association Task force on Practice Guidelines (Committee on Perioperative Cardiovascular Evaluation for Noncardiac Surgery). J Am Coll Cardiol 1996;27:910-48.

6. Bach DS, Eagle KA. Dobutamine stress echocardiography: stressing the indications for preoperative testing. Circulation 1997;95:8-10.

7. Poldermans D, Rambaldi R, Fioretti PM, Boersma E, Thomson IR, van Sambeek MRHM, et al. Prognostic value of dobutamineatropine stress echocardiography for perioperative and late cardiac events in patients scheduled for vascular surgery. Eur Heart J 1997;18(suppl D):D86-96.

8. Yock PG, Popp RL. Non invasive estimation of right ventricular systolic pressure by Doppler ultrasound in patients with tricuspid regurgitation. Circulation 1984;70:657-62.

9. Hatle L, Angelsen BAJ, Tromsodal A. Non-invasive estimation of pulmonary artery systolic pressure. Br Heart J 1981;45:157-65.

10. Kitabatake A, Inque M, Asao M, Masuyama T, Tanouchi J, Morita T, et al. Non invasive evaluation of pulmonary hypertension systolic pressure by pulsed Doppler technique. Circulation 1983;68:302-9.

11. Chan KL, Currie PJ, Seward JB, Hagler DJ, Mair DD, Tajik AJ. Comparison of three Doppler ultrasound methods in the prediction of pulmonary artery disease. J Am Coll Cardiol 1987;9:54954.

12. Armstrong WF. Stress echocardiography: introduction, history and methods. Prog Cardiovasc Dis 1997;6:499-522.

13. Bourdillon PDV, Broderick TM, Sawada SG, Armstrong WF, Ryan T, Dillon JC, et al. Regional wall motion index for infarct and non infarct regions after reperfusion in acute myocardial infarction: comparison with global wall motion index. J Am Soc Echocardiogr 1989;2:398-407.

14. Shaw LJ, Eagle KA, Gersh BJ, Miller DD. Meta-analysis of intravenous dipyridamole-thallium-201 imaging (1985 to 1994) and dobutamine echocardiography (1991 to 1994) for risk stratification before vascular surgery. J Am Coll Cardiol 1996;27:78798.

15. Poldermans D, Arnese M, Fioretti P, Boersma E, Thomson IR, Rambaldi R, et al. Sustained prognostic value of dobutamine stress echocardiography for late cardiac events after major noncardiac vascular surgery. Circulation 1997;95:53-7.

16. Eichelberger J, Schnarz K, Black E, Green R, Ouriel K. Medical value of dobutamine echocardiography before vascular surgery. Circulation 1992;86(Suppl):I789.

17. Lane RT, Sawada SG, Segar DS, Ryan T, Lalka SG, Williams R, et al. Dobutamine stress echocardiography for assessment of cardiac risk before non cardiac surgery. Am J Cardiol 1991;68:9767.

18. Lalka SG, Sawada SG, Dalsing MC, Cikrit DF, Sawchuk AP, Kovacs RL, et al. Dobutamine stress echocardiography as a predictor of cardiac events associated with aortic surgery. J Vasc Surg 1992;15:831-42.

19. Langan EM, Youkey JR, Franklin DP, Elmore JR, Costello JM, Nassef LA. Dobutamine stress echocardiography for cardiac risk assessment before aortic surgery. J Vasc Surg 1993;18:905-13.

20. Davilla-Roman VG, Waggoner AD, Sicard GA, Geltaman EM, Schechtman KB, Perez JE. Dobutamine stress echocardiography predicts surgical outcome in patients with an aortic aneurysm and peripheral vascular disease. J Am Coll Cardiol 1993;1:957-63.

21. Poldermans D, Arnese M, Fioretti PM, Thomson IR, Boersma E, van Urk H. Improved cardiac risk stratification in major vascular surgery with dobutamine-atropine stress echocardiography. J Am Coll Cardiol 1995;26:648-53.

22. Pellika PA, Roger VL, Oh JK, Seward JB, Tajik AJ. Safety of performing dobutamine stress echocardiography in patients with abdominal aortic aneurysm $\geq 4 \mathrm{~cm}$ in diameter. Am J Cardiol 1996;77:413-6.

23. Bates JR, Sawada SG, Segar DS, Spaedy AJ, Petrovic O, Fineberg NS, et al. Evaluation using dobutamine stress echocardiography in patients with insulin-dependent diabetes mellitus before kidney and/or pancreas transplantation. Am J Cardiol 1996;77:175-9.

24. Brenner M, Yusen R, McKenna R, Sciurba F, Gelb AF, Fischel R, et al. Lung volume reduction surgery for emphysema. Chest 1996;110:205-18.

25. Russi EW, Stammberger U, Weder W. Lung volume reduction surgery for emphysema. Eur Respir J 1997;10:208-18.

26. Cooper JD, Patterson GA, Sundaresan RS, Trulock EP, Yusen RD, Pohl MS, et al. Results of 150 consecutive bilateral lung volume reduction procedures in patients with emphysema. J Thorac Cardiovasc Surg 1996;112:1319-30.

27. McKenna RJ, Brenner M, Fischel RJ, Gelb AF. Should lung volume reduction for emphysema be unilateral or bilateral? J Thorac Cardiovasc Surg 1996;112:1331-9. 